\title{
EVALUACIÓN DE LA CALIDAD DE LA ATENCIÓN DE ENFERMERÍA EN EL HOSPITAL SANTA FE: UN ESTUDIO BASADO EN LA OPINIÓN DEL PERSONAL DE ENFERMERÍA, MEDICOS, PACIENTES Y FAMILIARES
}

\author{
Mtra Lasty Babeiro Almario", Mtra. Ma. Feresa Aychla Quinteri", Mtra Ma de los Angeies Portes Lagunos"**

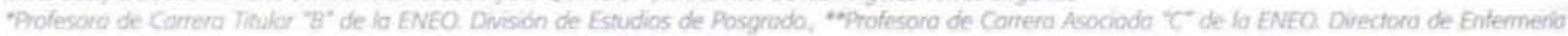 \\ del Hospitol Santa Fe, "wiprolesara de Camero Asociada "B" de lo ENEQ Sistemo Universidad Abierto
}

\section{RESUMEN}

En esta investigación se presenta la evaluación de la calidad de la atención de Enfermería, con base en la opinión del propio personal de Enfermeria, médicos, pacientes y familiares. Esta temática es importante porque la calidad de la atención es una prioridad en las instituciones de salud y educativas de nuestro país. La variable medida fue calidad la atención de Enfermeria, las dimensiones de esta variable fueron: estructura, proceso y resuitados. De cada dimensión se establecieron indicadores. El tipo de investigación fue descriptiva, transversal, analitica y de corte cuantitativo. En los criterios de inclusión se tomó en cuenta al personal de Enfermería, pacientes, médicas y familiares que estaban en el Hospital Santa Fé en los meses de julio, agosto y septiembre del 2005. Las técnicas e instrumentos de investigación utilizados fueron: fichas de trabajo, observación, entrevistas y cuestionarios. La muestra estudiada fue de 163 Enfermeras de los 3 tumos de trabajo, 127 pacientes, 119 médicos y 101 familiares. En los resultados se pudo constatar que la calidad de estructura, la calidad de proceso y la calidad de resultados del personal de Enfermeria en el Hospital Santa Fé es calificada por médicos, pacientes, familiares y el propio personal de Enfermería como de "buena a excelente" lo que refrenda al Hospital Santa Fé como el mejor hospital privado de México, por la calidad de sus servicios.

PALABRAS CLAVE. Calidad de la atención de Enfermeria, calidad de la atención en salud, calidad de estructura, calidad de proceso y calidad de resultados.

\section{ABSTRACT}

In this research, nursing care quality assessment is presented, based an the nursing stoff, physicians, patients, and families own opinion. The subject matter is impartant becouse attention quality is o priority for our country's health and education institutions. The measured variable was nursing care quality, and the dimensions for this voriable were: structure, process, and outcomes. Indicators were established from each dimension. The research was descriptive, transversal, analytical and quantitative. The nursing staff, patients, physicians, and relatives who were in Santa Fe Hospital throughout July, August, and September, 2005 accounted as inclusion critenia; research techniques and instruments used were: working cards, observation, interviews, and questionnaires. The studied sample included 163 nurses in the 3 work shifts, 127 patients, 119 physicians, and 101 rejatives. From the outcomes it could be confirm that structure quality, process quality, and nursing staff outcomes quality in Santa Fe Hospital is graded by physicians, potients, relatives, and the nursing staff itself as hoving a "good to excellent quality", which endorses Santa Fe Hospital as the best private hospital in Mexico due the quality of its services

KEY WORDS: nursing care quality, health care quality, structure quality, process quality, and outcomes quality. 


\section{INTRODUCCIÓN}

Enrique Ruelas y Julio Querol señalan que puede afirmarse con certeza que la desigualdad más grave del sistema de salud ya no se da exclusiva ni principalmente en al cobertura, sino en la calidad de los servicios que perciben los diferentes grupos sociales. "1) La calidad es pues, un derecho que debe ser garantizado por lo tanto, la garantía de la calidad es imperativa en los sistemas de salud y de manera concreta, en las organizaciones que los conforman. El presente articulo tiene por objeto dar a conocer la evaluación de la calidad de la atención de enfermería en el Hospital Santa Fé, con base en la opinión del propio personal de Enfermería, médicos pacientes y familiares.

Problema: Como pregunta eje de investigación se enuncó la siguiente: Como evalúan la calidad de la atención de Enfermeria, el propio personal de Enfermeria, los médicos, pacientes y familiares en el Hospital Santa Fé, en México, D.F.?

Objetivos: El objetivo general de la investigación fue evaluar la calidad de la atención de Enfermeria con base en la opinión de las propias Enfermeras, los médicos, pacientes y familiares. Como objetivos especificos se enunciaron los siguientes: Diagnosticar la problemática que tiene el Hosptal Santa Fé en las dimensiones de estructura, proceso y resultados de la calidad de la atencoón de Enfermería y proponer diversas medidas tendientes a mejorar la calidad de la atención de Enfermeria a las personas usuarias del Hospital Santa Fé.

Justificación: Hoy en dia la calidad de la atención es y debe ser una prioridad de las instituciones educativas y de salud, la certificación de los hospitales, el ISO 9000, el Premio Nacional de Calidad, etc., son tan solo algunos de los esfuerzos que se realizan para mejorar la calidad de la atención, sin embargo, es necesario para favorecer el proceso de mejora continua de la calidad en los servicios de salud. Es en este marco de la Cruzada Nacional por la Calidad de los Servicios de Salud 2001 - 2006 que el Cobiemo de México y el Convenio Interinstitucional firmado entre la Escuela Nacional de Enfermería y Obstetricia (ENEO) de la UNAM con el Hospital privado "Santa Fé" de la Ciudad de México es, que la presente investigación se justifica porque responde a las necesidades de investigación conjunta entre ambas instituciones en torno a la evaluación y a la mejora continua de la calidad del cuidado de Enfermeria en la atención integral de las personas usuarias de los servicios de salud. Para la ENEO esta investigación es relevante pues existe la necesidad de crear sedes de investigación con instituciones de salud públicas y privadas que propicien la mejora de la práctica del cuidado de Enfermería y que vinculen la docencia con los servicios asistenciales consolidando la investigación institucional que es uno de los objetivos prioritarios de la Escuela. Para el Hospital Santa Fé, esta investigación es importante pues desde el mes de mayo del 2002 en que se implementó el modelo de calidad y de mejora continua, no se ha llevado a cabo una evaluación del cuidado Enfermero para visualizar si la calidad de la atención de Enfermeria corresponde a las acciones planeadas. En dicho contexto, a tres años de iniciado el programa, se hace necesaria una evaluación que identifique si el nivel de calidad que actualmente tiene el Hospital garantiza el trato adecuado, genera satisfacción y provee una atención efectiva, eficiente, ética y segura acorde a las medidas implementadas y a la misión, visión y valores organizacionales de la Institución hacia la población usuaria.

Marco Teórico: Los cuidados de Enfermería a los pacientes son importantes y fundamentales en la conservación de la calidad asistencial. Sin embargo, a veces es dificil ponderar el impacto que estos cuidados de Enfermeria tienen en el total del servicio prestado por el centro o el equipo sanitario. Además de este primer enfoque de la relación de la calidad de los cuidados con la de los servi-

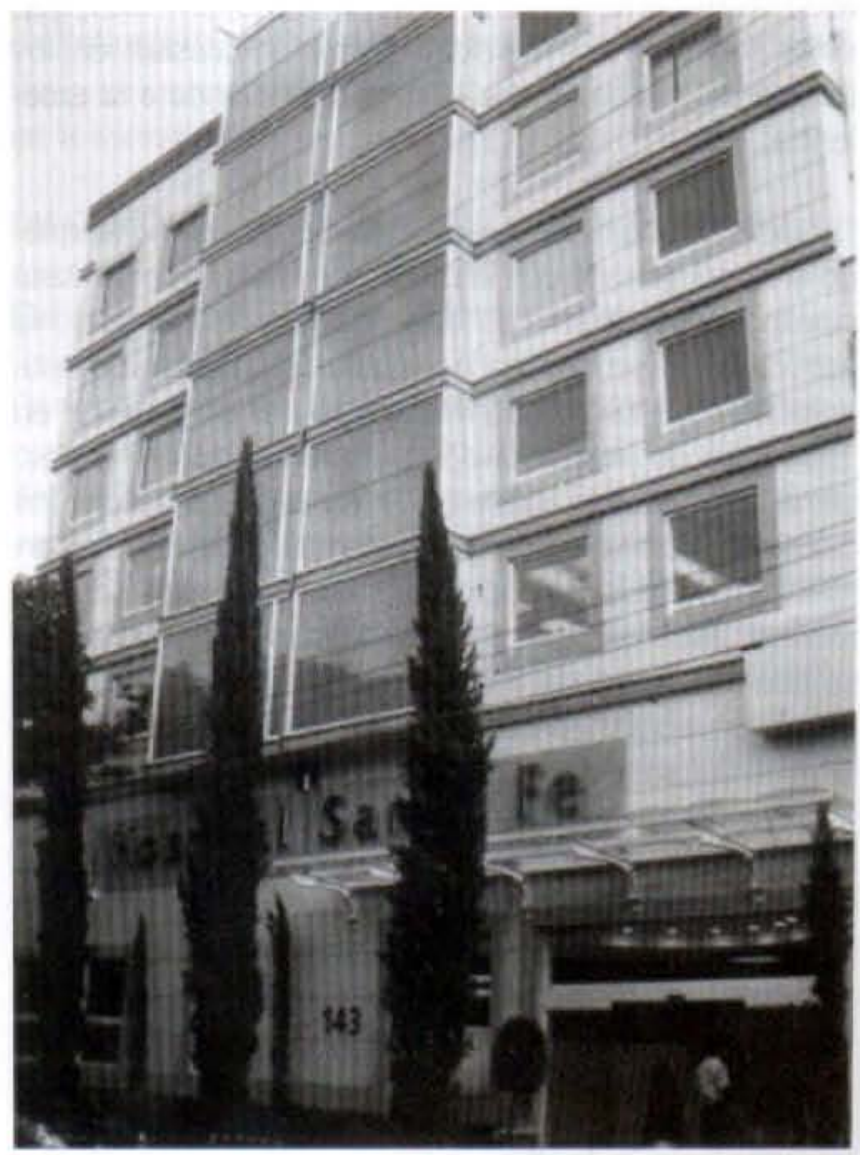


cios y con todo asistencial, es necesario abordar la posibilidad de identificar los aspectos que exige la consideración y valoración de la calidad en relación estrecha con el personal de Enfermería y sus cuidados ${ }^{(2)}$.

En primer lugar, la importancia de las acciones de Enfermería y sus consecuencias en el estado de salud de las personas usuarias, es decir el impacto que producen en el bienestar y la salud, hace patente la necesidad de controlar estas acciones y valorarlas en sí mismas garantizando su calidad que es determinante, tanto para el paciente o cliente, como para la institución. En segundo lugar, el volumen de trabajo y de las acciones de las Enfermeras supone un elevado porcentaje del total de la actividad de cualquier institución sanitaria. Ello hace preciso que las consideraciones de eficacia, adecuación y otros, se apliquen y muy especialmente a la labor que llevan a cabo estos profesionales. En tercer lugar, en las modernas instituciones de salud hay que tener en cuenta que cada vez más, las Enfermeras tienen la capacidad de planificar, ejecutar y evaluar sus acciones profesionales en un contexto de autonomía y responsabilidad profesional propias, en relación de cooperación e interdependencia con otros profesionales. Tal autonomía solo es posible si se acompaña de una seguridad y calidad en la acción que garantiza a los usuarios de los servicios Enfermeros, ya sean las personas usuarias o los otros profesionales, que estos servicios con identidad propia se prestan en los niveles de calidad idónea o incluso tendiendo a la excelencia.

Desde los trabajos de Avedis Donabedian, se ha aceptado la forma general que la calidad de la atención sanitaria puede evaluarse en tres aspectos que la integran: la estructura, el proceso y los resultados. Responde este enfoque cuya formulación se realizó por primera vez en 1956, siendo revisado posteriormente por el propio Donabedian y otros autores, a la necesidad de valorar los diferentes componentes o elementos de la calidad en función del análisis de los medios que se emplean, de los medios utilizados para prestar la atención o sea, el proceso que se sigue y del impacto producido en la situación de salud o enfermedad objeto de análisis que se presenta por resultados de la acción. Para Yanet Griffith y Paula J. Christensen, el proceso y el resultado pueden ser subdivididos en dos categorias concurrentes (presente) y retrospectiva (pasada). Asi, calidad de la atención de Enfermería también se mide en tres dimensiones: estructura, proceso y resultado (3). Los indicadores de la dimensión de "estructura" en Enfermería son: plantilla personal, dotación de artículos de consumo, conocimiento de las políticas y reglamentos institucionales, relaciones humanas, organización del trabajo de Enfermería, revisión del expediente clínico, sistemas de información de Enfermería, distribución en el área de trabajo y recursos fisicos para el trabajo. De hecho, la estructura se dirige a las instalaciones físicas, equipos y patrón de organizaciones. La dimensión de "proceso" se centra en las actividades de Enfermería. Estas actividades pueden juzgarse observando el rendimiento, preguntando en los pacientes o revisando las notas de Enfermeria en las hojas de evolución, la evaluación concurrente del proceso examina el rendimiento de la Enfermería en el momento en que tiene lugar.

La evaluación retrospectiva del proceso es implementada después de que el paciente ha sido dado de alta. La dimensión de proceso tiene los siguientes indicadores: planeación de actividades, ejecución del proceso de atención de Enfermería, conocimiento del trabajo de Enfermería, conocimientos de las responsabilidades de Enfermeria, habilidades y destrezas en el trabajo, calidez humana, habilidad para la enseñanza de Enfermeria, habilidad para el mejoramiento del trabajo y capacidad profesional de Enfermería. La dimensión de "resultados" se centra en los cambios de conducta y el estado de salud del paciente, la Enfermera busca la evidencia de la mejoría, en el estado de salud que resulta de su intervención. La evaluación concurrente del resultado juzga la capacidad del paciente para demostrar programas de conducta medibles en su estado de salud, sus destrezas o su conocimiento. La dimensión de resultados se puede medir en Enfermeria con los siguientes indicadores: satisfacción de las necesidades del cliente, promedio de dias estancia, diagnóstico oportuno, tratamiento adecuado, presencia de iatrogenias, altas voluntarias, reporte del paciente y rapidez en la atención entre otros. Para Avedis Donabedian, la piedra angular de la calidad en la atención a la salud y particularmente en Enfermería, es contar con profesionales bien preparados y con una capacitación adecuada. Para ello es necesario que los conocimientos básicos se renueven constantemente introduciendo lo nuevo y descontando lo absoluto. Así, cada profesional de la salud debería aprender los principios y los métodos para la garantía de la calidad (4).

\section{METODOLOGIA.}

La variable medida fue: calidad de la atención de Enfermería. Los indicadores de "estructura" para medir esta variable fueron: Dotación de mobiliario y equipo medico, distribución física de los espacios, funcionamiento del equipo electromédico, cantidad de factores humanos, suficiente dotación de medicamentos, suficiente. 
dotación de ropa, alimentos que consumen los pacientes y medidas de seguridad para los pacientes. Los indicadores de "proceso" fueron: Rutinas de Enfermeria, cumplimiento cabal de las actividades, técnicas de Enfermeria, relación Enfermera-paciente, trato humano, comunicación e información de Enfermeria a los pacientes, calidez en la atención, dominio de las técnicas y procedimientos y respecto al personal de Enfermería a los pacientes. Los indicadores de "resultado" fueron: satisfacción de los pacientes, promedio de dias estancia, diagnósticos oportunos para los tratamientos, tratamientos adecuados, existencia de errores de Enfermeria (iatrogenias) altas voluntarias y rapidez en la atención.

Tipo y diseño de investigación: El tipo de investigación fue descriptivo, transversal, analitico y de corte cuantitativo. El estudio identifica, describe y evalúa el nivel de calidad de la atención de Enfermeria en un momento determinado, en los meses de julio, agosto y septiembre del 2005. La investigación se diseñó en tres etapas: En la primera, se estructuró el proyecto, el estado del arte sobre la calidad de la atención de Enfermeria y se adoptó el instrumento al Hospital Santa Fé, realizando para ello, una prueba piloto. En la segunda etapa, se realizó la recolección, procesamiento y análisis de los datos y en la tercera etapa se integró el informe de investigación.

Los criterios de inclusión, exclusión y eliminación:

En los criterios de inclusión y exclusión: se tomó en cuenla al personal de Enfermeria, los pacientes, los médicos y los familiares que estaban en el Hospital Santa Fé durante los meses de julio, agosto y septiembre del 2005, fechas en que se aplicó la encuesta. En el criterio de exclusión se tomó en cuenta al personal de Enfermería, médicos, pacientes y familiares que son de otras instituciones ajenas al Hospital Santa Fé. También se excluyó a camilleros y secretarias. En el criterio de eliminación se tomó en cuenta a aquellos Enfermeros, médicos, pacientes y familiares que no aceptaron contestar los cuestionarios, o bien que dejaron los cuestionarios incompletos.

Técnicas e instrumentos de recolección: Las técnicas de investigación utilizadas fueron: fichas de trabajo, observación, entrevista y cuestionarios. Con las fichas de trabajo fue posible ordenar y clasificar la información relativa al marco teórico y referencial de la variable calidad de la atención de Enfermería; a través de la observación se pudieron obtener las diversas impresiones y actitudes del personal de Enfermeria, pacientes, médicos y familiares en el momento de la encuesta, con la entrevista se obtuvo los testimonios orales de las entrevistadas asi como sus impresiones en relación con la calidad de la atención de Enfermería que se brinda con el Hospital Santa Fé. Los cuestionarios fueron el principal instrumento de recolección de los datos. Se utilizaron 4 cuestionarios, uno para el personal de Enfermeria, otro para médicos, otro para pacientes y otros para familiares. Se estructuró cada cuestionario con 27 preguntas, 2 de datos generales, y 25 que median la calidad de la atención de Enfermería, todas las respuestas de los cuestionarios eran cerradas estructuradas utilizando el sistema Likert con cinco opciones de respuesta, desde la mejor, hasta la peor opción.

Población y muestra: La población de estudio fueron 200 Enfermeras, de los diferentes turnos, 300 médicos, 300 pacientes y 200 familiares. La muestra estudiada fueron 163 enfermeras, 127 pacientes, 119 médicos y 101 familiares. Esto representa para las Enfermeras el 58.12\% del universo, para los médicos el $21.63 \%$, para los pacientes el $21.16 \%$ y para los familiares el $25.25 \%$ del universo.

Consideraciones éticas: Para este estudio se consideró el principio del anonimato y privacidad del reglamento de la Ley General de Salud en materia de investigación para la salud, titulo $2^{\circ}$, cap. 1, Art. 10, Fracc. $V$ que se refiere al consentimiento informado de los sujetos implicados en una investigación y el principio de privacidad del Art. 16 que hace referencia al anonimato de los sujetos de investigación, por lo que no se solicitó el nombre al personal de Enfermeria, médicos, pacientes y familiares entrevistados y se les respetó su libertad de decidir participar o no en la investigación.

\section{DISCUSIÓN.}

\section{Calidad de estructura en el Hospital Santa Fé.}

En relación a si existe suficiente dotación de mobiliario y equipo electromédico en el Hospital Santa Fé, el 58.90\% de las Enfermeras coinciden que "la mayoria de las veces" si hay suficiente dotación de material y equipo para el trabajo de Enfermería, en tanto, el $74.02 \%$ de los pacientes dicen que siempre ha habido suficiente dotación de material y equipo para trabajar con los pacientes, el $60.50 \%$ de los médicos y el $67.33 \%$ de los familiares consideran que siempre ha habido suficiente dotación de mobiliario y equipo médico. Llama la atención que los pacientes, médicos y familiares califican y coinciden en sus respuestas, en tanto las Enfermeras tienden a disminuir su calificación considerando como si la "mayoría de las veces" cuentan con la dotación de material y equipo para el trabajo de Enfermeria. En torno a la distribución física de los espacios en el Hospital el $45.39 \%$ del personal de Enfermería la considera como "regular", en tanto 
que el $45.68 \%$ de los pacientes la considera como "excelente", el $54.63 \%$ de los médicos como "buena" al igual que el $52.48 \%$ de los familiares. De hecho, coinciden en las respuestas de que la distribución es "buena" los médicos y familiares. En torno a si existe suficiente cantidad de factores humanos de Enfermeria para el trabajo en el Hospital, el $50.93 \%$ del personal de Enfermería considera que a veces si hay personal de Enfermería, pero otras veces falta este personal especialmente los dias festivos. En cambio, el $49.89 \%$ de los pacientes, el $52.95 \%$ de los médicos y el $60.40 \%$ de los familiares coinciden en afirmar que en este Hospital siempre hay suficiente cantidad de personal de Enfermeria en atención de los pacientes. Esto demuestra el esfuerzo que hace la Jefatura de Enfermeria para cubrir las vacantes y faltantes de personal de Enfermeria y ofrecer siempre la misma calidad de atención en el Hospital.

En relación a si hay suficiente dotación de medicamentos para todos los pacientes, el 37.42\% de las Enfermeras consideran que la "mayoria de las veces" si hay medicamentos que se requieren para la atención de los pacientes, en tanto que el $72.45 \%$ de los pacientes, el $67.23 \%$ de los médicos y el $83.17 \%$ de los familiares coinciden en que siempre hay suficiente dotación de medicamentos para todos los pacientes. En cuanto a si existe suficiente dotación de ropa para los pacientes en el Hospital, el $47.23 \%$ de las Enfermeras consideran que "la mayoria de las veces" si cuentan con suficiente cantidad de ropa para los pacientes, en tanto que el $69.30 \%$ de los pacientes, el $84.88 \%$ de los médicos y el $78.22 \%$ de los familiares coinciden en que el Hospital siempre hay suficiente dotación de ropa para los cambios que necesitan los pacientes. Una vez más las Enfermeras califican más bajo la existencia de ropa en el Hospital, aunque los médicos, los pacientes y familiares dan una calificación de "excelente" en este rubro. En cuanto a las medidas de seguridad para los pacientes del Hospital el $49.70 \%$ de las Enfermeras consideran que "la mayoría de las veces" si existen medidas de seguridad para los pacientes. De igual forma piensan el $50.43 \%$ de los médicos y el $57.49 \%$ de los familiares. Por su parte, el $58.42 \%$ de los familiares consideran que en el Hospital siempre ha habido las medidas de seguridad que permite que los pacientes estén protegidos. De hecho, para este rubro coinciden Enfermeras, pacientes y médicos. Los familiares califican más alto las medidas de seguridad.

En términos generales se puede considerar que para las Enfermeras la calidad de estructura del Hospital Santa Fé es considerada como "buena". Las calificaciones más altas de la dimensión de estructura la dan las Enfermeras a la dotación de equipo electromédico, al funcionamiento del equipo, a la dotación de medicamentos, a la existencia de ropa y alimentos que consumen pacientes. Las calificaciones más bajas de estructura que dan las Enfermeras están en: la distribución física de espacios del Hospital y la insuficiente cantidad de personal de Enfermeria. Por el contrario, médicos, pacientes y familiares consideran la calidad de estructura del Hospital como de "buena" a "excelente".

\section{Calidad de proceso en el Hospital Santa Fé.}

En relación con la comunicación e información de Enfermería para los pacientes, el $49.08 \%$ de las Enfermeras considera que la comunicación e información con los pacientes es "buena" ya que las Enfermeras si informan a los pacientes todas aquellas cuestiones relacionadas con su tratamiento, también en esto coinciden el $55.12 \%$ de los pacientes, el $45.38 \%$ de los médicos y el $47.53 \%$ de los familiares. En cuanto a la calidez en al atención de Enfermería a los pacientes, el $47.85 \%$ de las Enfermeras piensan que "la mayoria de las veces" el personal de Enfermeria si es cálido con los pacientes. De igual forma, el $58.26 \%$ de los pacientes consideran que "la mayoria de las veces" el personal de Enfermeria si es cálido en su trato para con los pacientes. Por el contrario, el $58.83 \%$ de los médicos y el $54.46 \%$ de los familiares coinciden en que el personal de Enfermeria es muy cálido en su trato para los pacientes. Esto significa que la calidez del personal de Enfermeria está mejor valorada por médicos y familiares que por el propio personal de Enfermeria y pacientes. En relación a si las Enfermeras dominan las técnicas en el Hospital, el $60.26 \%$ de las Enfermeras consideran que si las dominan. De igual forma, el $62.20 \%$ de los pacientes opinan lo mismo, aunque el $47.07 \%$ de los médicos y el $53.47 \%$ de los familiares creen que las Enfermeras tienen un "excelente" dominio de las técricas y procedimientos de Enfermeria para brindar calidad de atención a los pacientes. Llama la atención que los médicos y los familiares califican de "excelente" las técnicas y procedimientos de Enfermería que las propias Enfermeras que las califican como "buenas". En cuanto a si existe personal de Enfermería capacitado en el Hospital, el $42.74 \%$ de las Enfermeras piensan que "a veces" el personal de Enfermería sí está capacitado, pera otras veces les falta capacitación. En tanto, el $48.05 \%$ de los pacientes, el $57.99 \%$ de los médicos y el $54.46 \%$ de los familiares coinciden en que en el Hospital todo el personal de Enfermeria está capacitado y adiestra. do para brindar la más alta calidad de atención de Enfermería a los pacientes. Aquí el propio personal de Enfermeria se califica de "regular" en la capacitación, 
en tanto que pacientes, médicos y familiares lo califican de "excelente". Esto demuestra la honestidad del personal de Enfermería quien reconoce sus deficiencias en materia de capacitación. A pesar de ello, demuestra ante los pacientes la suficiente cantidad de conocimientos en técnicas y procedimientos.

En relación a cómo consideran las rutinas de Enfermería en el Hospital, el $57.67 \%$ de las Enfermeras creen que las rutinas son "buenas" y esto permite que los pacientes estén satisfechos, el $48.74 \%$ de los médicos y el $47.50 \%$ de los familiares piensan igual que las Enfermeras. Solo los pacientes en un $51.96 \%$ califican de "excelentes" las rutinas de Enfermeria ya que el personal trabaja coordinadamente en la atención a los pacientes. En cuanto a si las Enfermeras cumplen cabalmente con sus actividades, el $54.60 \%$ del personal de Enfermería manifiesta que "la mayoria de las veces" si cumple con las actividades y lo mismo consideran los pacientes. En tanto el $49.59 \%$ de los médicos y el $56.44 \%$ de los familiares creen que el personal de Enfermería si cumple cabalmente con todas sus actividades encomendadas en la atención de los pacientes. De hecho, los médicos y familiares califican mejor al personal de Enfermería que las propias Enfermeras del hospital. En cuanto a la relación Enfermera-Paciente, el personal de Enfermería considera en un $60.13 \%$ que la relación es "buena" ya que hay respeto y buen trato. Esto mismo, consideran en un $51.99 \%$ los pacientes. Por el contrario, el $51.27 \%$ de los médicos y el $57.43 \%$ de los familiares, piensan que la relación es "excelente" ya que existe comunicación y respeto en el trato a los pacientes. En relación a si existe trato humano del personal de Enfermería los pacientes, el 52.15\% de las Enfermeras creen que si hay trato humano y eso hace que los pacientes se sientan bien. De igual forma, el $50.39 \%$ de los pacientes piensan igual que las Enfermeras. En tanto, el $57.99 \%$ de los médicos y el $57.43 \%$ de los familiares creen que el trato es "excelente" por parte del personal de Enfermeria. En torno al respeto del personal de Enfermería hacia los pacientes, el $61.36 \%$ del personal considera que siempre respeta a los pacientes. En esto coinciden el $48.04 \%$ de los pacientes, el $74.79 \%$ de los médicos y el $60.40 \%$ de los familiares.

En general, la calidad de proceso es considerada por el personal de Enfermería como de "buena" a "excelente". Las mejores calificaciones que dan las Enfermeras a la calidad de proceso están en: respeto a los pacientes, elaboración de rutinas de Enfermeria, desarrollo de técnicas de Enfermeria y cumplimiento cabal de actividades. La calificación más baja que dan las Enfermeras está en la capacitación y adiestramiento del personal. De igual forma, los pacientes, los médicos y los familiares otorgan calificaciones altas a la calidad del proceso de atención de Enfermería calificándola como de "buena" o "excelente" calidad.

\section{Calidad de resultados en el Hospital Santa Fé.}

En relación a si los pacientes están satisfechos con la atención que Enfermería les proporciona, el $52.76 \%$ de las Enfermeras dicen que sí están satisfechos, el 59.05\% de los pacientes consideran que ellos están muy satisfechos con la atención que Enfermeria les proporciona, por ello se sienten bien en su estancia en el Hospital, el $49.58 \%$ de los médicos y el $60.40 \%$ de los familiares coinciden con los pacientes. Esto significa que pacientes, médicos y familiares consideran estar muy satisfechos con la atención de Enfermeria, en tanto las Enfermeras creen que están satisfechos "a veces". En relación con el promedio de días estancia hospitalaria todas las respuestas coinciden en que el promedio de dias estancia es de entre 3 y 5 dias, ya que la recuperación de los pacientes es rápida. Para el $52.15 \%$ de las Enfermeras, el $77.96 \%$ de los pacientes, el $73.11 \%$ de los médicos y el $44.56 \%$ de los familiares el promedio de dias estancia hospitalaria es de 3 a 5 dias. En torno a si los pacientes del Hospital Santa Fé reciben diagnósticos oportunos en sus tratamientos, el $71.66 \%$ de las Enfermeras, el $58.83 \%$ de los médicos, el $71.65 \%$ de los pacientes y el $70.30 \%$ de los familiares coinciden en que efectivamente los pacientes si reciben los diagnósticos oportunos y gracias a ello se les puede atender rápidamente. En relación a si los tratamientos de los pacientes son los adecuados, el $54.60 \%$ del personal de Enfermería creen que los tratamientos de los pacientes son los adecuados y se obtienen buenos resultados, en cambio para el $74.02 \%$ de los pacientes, el $66.40 \%$ de los médicos y el $72.28 \%$ de los familiares los tratamientos que se les dan a los pacientes sí son los adecuados y esto permite su pronta recuperación.

En cuanto a si consideran que existen iatrogenias causadas por médicos o Enfermeras se obtuvo que el $34.97 \%$ del personal de Enfermería creen que a veces cuando hay descuidos, si existen errores que pueden llamarse iatrogenias. Por el contrario, el $73.23 \%$ de los pacientes, el $36.14 \%$ de los médicos y el $66.34 \%$ de los familiares coinciden en que en el Hospital no hay ninguna iatrogenia causada por errores médicos o de Enfermería. Desde luego, la no presencia de iatrogenias en el Hospital refuerza aun más la satisfacción de los pacientes y garantiza la calidad de atención. Otro indicador de resultados fue la rapidez de la atención de Enfermería a los pacientes. Las respuestas que se obtuvieron fueron que el 
$44.89 \%$ del personal de Enfermería creen que la atenciòn es rápida, ya que la Enfermería "la mayoria de las veces" acude con prontitud. Esta misma opinión la externaton el $50.43 \%$ de los médicos, el $44.89 \%$ de los pacientes y el 52.4800 de los familiares.

En términos generales, la calidad por los resultados fue calificada como "buena" por el personal de Enfermeria en los siguientes indicadores: satisfacción de los pacientes por la atención de Enfermeria recibida, diagnósticos oportunos, tratamientos adecuados, altas voluntarias y atención rápida. La única calificación de "regular" del personal de Enfermeria fue en la presencia de iatrogenias. Por el contrario, las Enfermeras calificaron de "excelente" la rapidez en la atención.

Por su parte, los pacientes, médicos y familiares coincidieron en que los paciente están muy satisfechos con la atención de Enfermería, con el promedio de dias estancia hospitalaria que es de 3 a 5 días, con los diagnósticos oportunos, con los tratamientos de los pacientes, con la no presencia de iatrogenias y con la no existencia de altas voluntarias en el Hospital. De lo anterior se puede decir que la evaluación de la calidad es "buena" para el personal de Enfermerla y es "excelente" para médicos, familiares y pacientes.

\section{CONCLUSIONES.}

Se lograron los objetivos de la investigación al poder evaluar la calidad de la atención de Enfermeria que se brinda en el Hospital Santa Fé, con base en la opinión del propio personal de Enfermeria, médicos, pacientes y familiares. Se pudo obtener que el $68.72 \%$ del personal de Enfermeria y el $49.50 \%$ de familiares, consideran que la calidad de la atención de Enfermerla es buena, en tanto que el $55.91 \%$ de pacientes y el $59.67 \%$ de los médicos opinan que la calidad de la atención de Enfermería es excelente. De hecho, globalizando las opiniones, el $95.28 \%$ de Enfermeras, médicos, pacientes y familiares opinan que la calidad de atención es de "buena a excelente". En relación con los objetivos especificos en donde se solicitaba diagnosticar la dimensión de estructura, proceso y resultados de la calidad de la atención de Enfermeria, se pudo obtener lo siguiente:

\section{Calidad de Estructura.}

El $92.95 \%$ de Enfermeras, pacientes, médicos y familiares consideran que en el Hospital Santa Fé siempre ha habido suficiente dotación de mobiliario y equipo médico, el 71.57\% piensan que la distribución física de los espacios en el Hospital es buena ya que dichos espacios

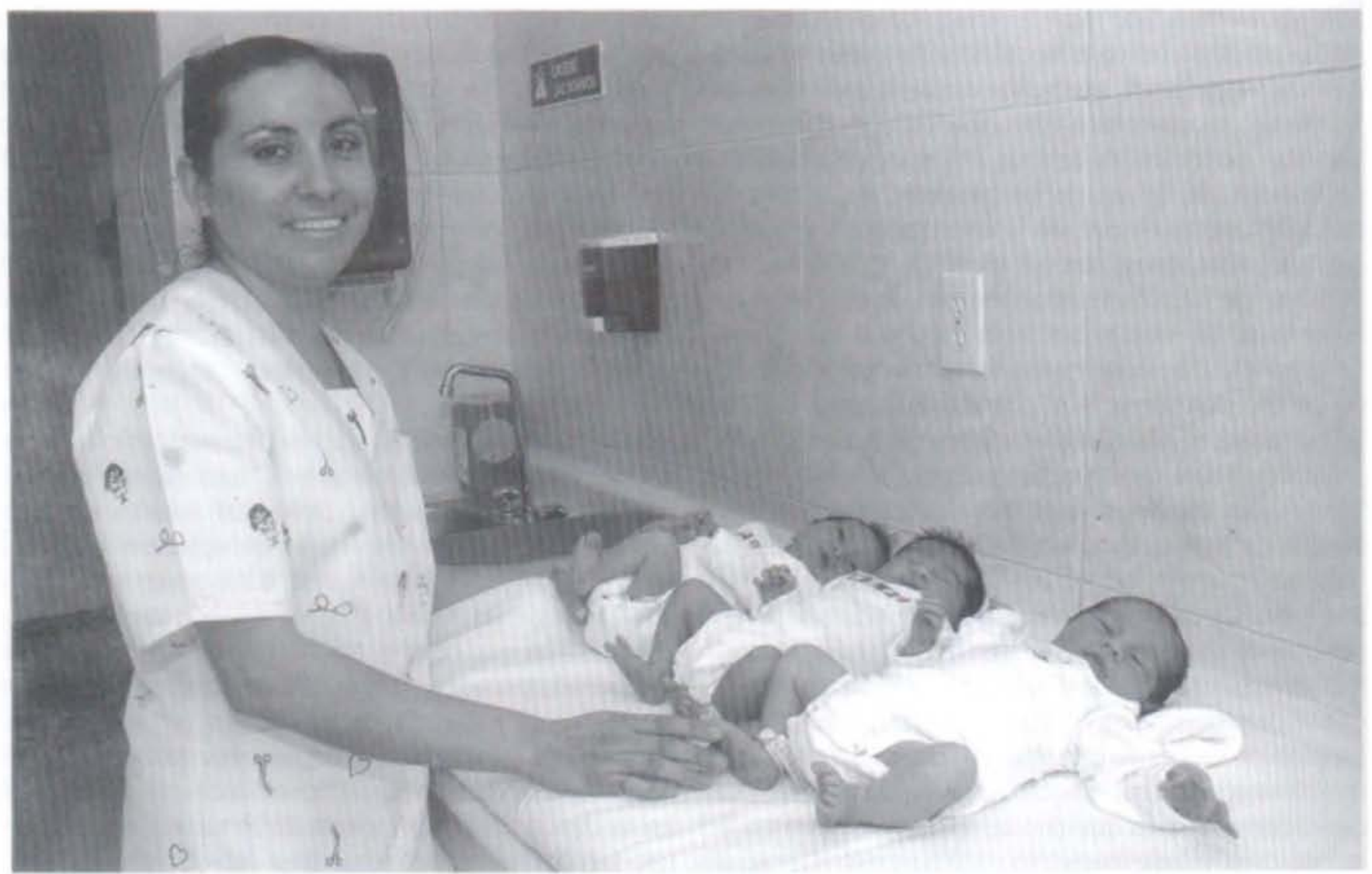


son amplios y se pueden transitar, el $85.30 \%$ manifiestan que el funcionamiento del equipo electromédico es bueno ya que los aparatos funcionan sin problema y el $73.15 \%$ dicen que siempre hay suficiente cantidad de personal de Enfermeria para la atención de los pacientes.

En otros hallazgos se pudo constatar que el $84.52 \%$ de las Enfermeras, médicos, pacientes y familiares consideran que en el Hospital siempre hay suficiente dotación de medicamentos para todos los pacientes, con lo que es posible realizar sus tratamientos, el $89.79 \%$ piensan que siempre hay suficiente dotación de ropa para los cambios que necesitan los pacientes, el $66.69 \%$ consideran que los alimentos son de excelente calidad y cantidad y el 89.04\% creen que en el Hospital siempre ha habido medidas de seguridad para los pacientes. Asi, la dimensión de estructura en el Hospital Santa Fé fue evaluada como de "buena a excelente".

\section{Calidad de Proceso}

El $87.67 \%$ de las Enfermeras, los médicos, los pacientes y los familiares consideran que las rutinas de Enfermería son de "buenas a excelentes" y esto permite tener satisfechos a los pacientes, el $86.66 \%$ piensan que las Enfermeras cumplen cabalmente con todas las actividades encomendadas en la atención de los pacientes, el 93.94\% creen que la relación Enfermera-Paciente es de buena a excelente y el $93.15 \%$ opinan que el personal de Enfermeria si da un trato humano a los pacientes. El $80.21 \%$ de las Enfermeras, médicos, pacientes y familiares creen que la comunicación e información que da el personal de Enfermeria a los pacientes va de buena a excelente; el $91 . \%$ trato a los pacientes, el $86.67 \%$ consideran que las Enfermeras si dominan las técnicas y procedimientos que desarrolla en el trabajo con los pacientes y el $94.52 \%$ opinan que el personal de Enfermería siempre respeta los pacientes.

Asi la dimensión de proceso en el Hospital Santa Fé fue evaluada como de "buena a excelente". De hecho, las calificaciones que miden "proceso" fueron más altas que la dimensión de "estructura", lo que da idea que los médicos, pacientes y familiares han sabido valorar el trabajo que Enfermeria desempeña en el Hospital.

\section{Calidad de Resultados}

El $88.42 \%$ del personal de Enfermeria, médicos, pacientes y familiares consideran que los pacientes están muy satisfechos con la atención que Enfermeria les proporciona, por ello se sienten muy bien en su estancia en el Hospital y el
$79.62 \%$ piensan que el promedio de estancia de los pacientes es de 3 a 5 dias, el $90.18 \%$ creen que los pacientes si reciben los diagnósticos oportunos para sus tratamientos y el $88.06 \%$ creen que los tratamientos de los pacientes en el Hospital, son los adecuados. El 70.01\% de las Enfermeras, médicos, pacientes y familiares consideran que no existen iatrogenias en el Hospital, el $84.50 \%$ piensan que las altas de los pacientes se dan cuando estos están recuperados y satisfechos de la atención recibida y el $84.72 \%$ opinan que la atención de Enfermeria es muy rápida ante los llamados de los pacientes.

Asi la dimensión de resultados fue evaluada en el Hospital Santa Fé de "buena a excelente". Por ello, el $95.28 \%$ de Enfermeras, pacientes, médicos y familiares manifiestan que la calidad de la atención de Enfermería es de buena a excelente.

Por último, a partir de la medición realizada de calidad de la atención de Enfermeria en sus dimensiones de : Estructura, Proceso y Resultados, los alumnos becarios de la ENEO, que realizan sus prácticas en el Hospital Santa Fé, podrán a partir de los indicadores medidos, proponer nuevos indicadores de la atención de Enfermería, realizar otras mediciones y comparar resultados, para favorecer su aprendizaje en materia de calidad. Con ello, no solo podrá ampliar su marco conceptual y referencial en calidad hospitalaria sino que también mejorarán sus destrezas en materia de investigación.

\section{RECOMENDACIONES}

\section{De estructura}

Acostumbrar al personal de Enfermeria a las palabras: evaluación, aseguramiento de la calidad, calidad total, mejora continua, que al internalizarlas resultará benéfico como estrategia de mejoramiento continuo.

Mantener una dotación suficiente y oportuna de medicamentos, ropa, material y equipo, para evitar la escasez de estos insumos en los servicios de Enfermeria y evitar se vea limitada la continuidad de la atención.

Fortalecer la capacidad técnica de las Enfermeras mediante la capacitación continua y el adiestramiento en servicio, para que este personal brinde siempre una mejor calidad de atención.

Incrementar el número de profesionales de Enfermería a fin de cubrir las necesidades de personal de los servicios y eficientar la atención de Enfermería a los usuarios. 
Evaluar continuamente los procesos de mejora continua en el Hospital para garantizar la satisfacción del usuario.

Detectar los errores o desviaciones en la atención del personal de Enfermería, mediante un control de calidad, para garantizar la mejora en la atención a los pacientes. Con ello se logrará también que todo el personal mantenga niveles elevados de competencia.

Realizar estudios de impacto de la atención del personal de Enfermería como: Licenciados, Pasantes, Becarios, Enfermeras Generales, que propicien la contratación de aquellos que tengan mejor impacto.

Reconocer de manera continua el trabajo del personal de Enfermeria, motivándolo a que siga desarrollándose y creciendo tanto de manera personal como profesional en beneficio de su persona y del Hospital.

Propiciar programas de introducción al puesto del personal de Enfermería, que garanticen la calidad del manejo de técnicas y procedimientos de Enfermeria en beneficio de los pacientes.

Fortalecer el respeto y la cooperación y la consideración entre el propio personal de Enfermería para que el trabajo de equipo se realice en un ambiente positivo y agradable entre pares.

Dar a conocer el personal de Enfermería el modelo de calidad que se lleva en el Hospital ubicando la participación tan importante del personal de Enfermeria dentro de ese modelo.

Propiciar un sistema de cultura organizacional hacia la calidad de la atención de Enfermería para que los comportamientos, conductas, hábitos y valores del personal de Enfermería, se oriente hacia la mejora continua cada día.

\section{De Proceso}

Fortalecer un trato oportuno, digno e integral a los usuarios que redunde en la mejora continua de la calidad de la atención.

Propiciar que el personal de Enfermería tenga mayor conocimiento de los pacientes, no solo en el aspecto de su padecimiento, sino en cuanto a sus expectativas presentes y futuras para propiciar una mejor y mayor relación Enfermera-Paciente.

Favorecer la atención integral de las personas usuarias que asisten al Hospital Santa Fé a través de promover una atención continua, personalizada y oportuna que garantice una pronta atención.

Fortalecer el trato humano del personal de Enfermería hacia los pacientes y familiares a través de cursos y seminarios de "Trato digno" y Trato humanizado".

Fortalecer el programa de Educación Continua de Enfermería para mejorar la capacitación en aspectos técnicos, disciplinarios, éticos y de derechos humanos, que favorezcan la eficiencia y la eficacia de la atención.

Diseñar un programa de alto desempeño del personal de Enfermería a partir de la evaluación individual y grupal así como la información que dan los pacientes, para reconocer y compensar al personal de Enfermería destacado por la gran responsabilidad en la atención a los pacientes.

Establecer una comunicación directa, continua y permanente con las personas usuarias con objeto de informar de manera precisa y permanente respecto de las dudas que tenga respecto de su atención durante su estancia en el Hospital.

\section{De Resultados}

Evaluar continuamente el grado de satisfacción de los usuarios que permita retroalimentar la atención de Enfermeria y brindar servicios de Enfermeria acorde a las expectativas de los pacientes.

Evaluar el grado de satisfacción o insatisfacción de los pacientes con respecto del servicio de Enfermería otorgado durante su estancia hospitalaria y obtener información oportuna para acciones correctivas y de retroalimentación en beneficio de los pacientes y del personal de Enfermeria.

Evaluar la satisfacción del personal de Enfermeria en el trabajo, identificando educación, capacitación y desarrollo, para que el personal encuentre-un mayor sentido en el trabajo, oportunidades de desarrollo y realización personal. Esta evaluación puede incluir tópicos como liderazgo, oportunidades de desarrollo, capacitación, ambiente de trabajo, cooperación, trabajo en equipo, comunicación, seguridad, reconocimientos, motivación, recursos para el desempeño de su función, entre otros.

Propiciar servicios de apoyo al personal de Enfermeria para mantenerlo satisfecho, con un programa de Desarrollo Social para las Enfermeras en donde se pueda 
considerar: guarderias, deportes, actividades sociales y culturales, transporte, alimentación en horarios de trabajo y preparación para el retiro.

Dar a conocer al personal de Enfermería cada año, los resultados de los siguientes valores institucionales: usuarios, mercados, competencia, comunidad hospitalaria e imagen institucional, desempeño favorable o desfavorable de Enfermeria, para involucrarlas cada dia más con la misión y visión del Hospital Santa Fé. Es decir, involucrarlás con la cultura de la calidad y de la mejora continua.

Evaluar periódicamente en los servicios de Enfermeria si el trato que se brinda a las personas usuarias favorece la dignificación de éstas y considera aspectos tales como: disponibilidad en la atención sin rechazos, el escuchar a los pacientes, resolver sus dudas, favorecer la disminución de los tiempos de espera, etc.

Contar con mecanismos que permitan monitorizar de manera continua que la atención sea integral, pronta y oportuna para garantizar la calidad del cuidado de Enfermeria en los diversos servicios asistenciales donde se tenga trato directo con los pacientes.

\section{REFERENCIAS BIBLIOGRÁFICAS}

Ruelas, Enrique y Querol J. En Armando Martinez Ramirez y Cols. Hocia una estrategia de garantia de colidad; satisfocción en la utilzoción de los servicios médicos. En la Revista Salud Pública de México, No. 12, Vol. 3, Julio-Septiembre. Rio de Janeiro, 1996, p. 400.

Mompart G., Mary Paz. Calidad de la atención de Enfermeria y de los cuidodos de Enfermeria. En Administración de los servicios de Enfermeria. Ed. Mason, Baceriona, 1995, p. 160

Griffith, Janet y Paula Christensen. Proceso de Atención de Enfermeria. Aplicación de Teorias, Gulas y Modelos. Ed. Manual Moderno. México 1993. p. 198.

Donabedian, Avedis, Garantio de la colidad, Quien es el responsable? En la Antologia Gerencias de los servicios de Enfermeria. Ed. ENEOUNAM. México, 1997. p. 393.

\section{DIRECCIÓN PARA CORRESPONDENCIA}

Mtra. Lasty Baiseiro Almario: lastybals@hotmail.com

Mtra. Ma. Teresa Ayala Quintero: tayala@hopitalsantafe.hotmail.com.mx Mtra. Ma. de los Angeles Torres Lagunas:

angeles_torres2000@yahoo.com.mx

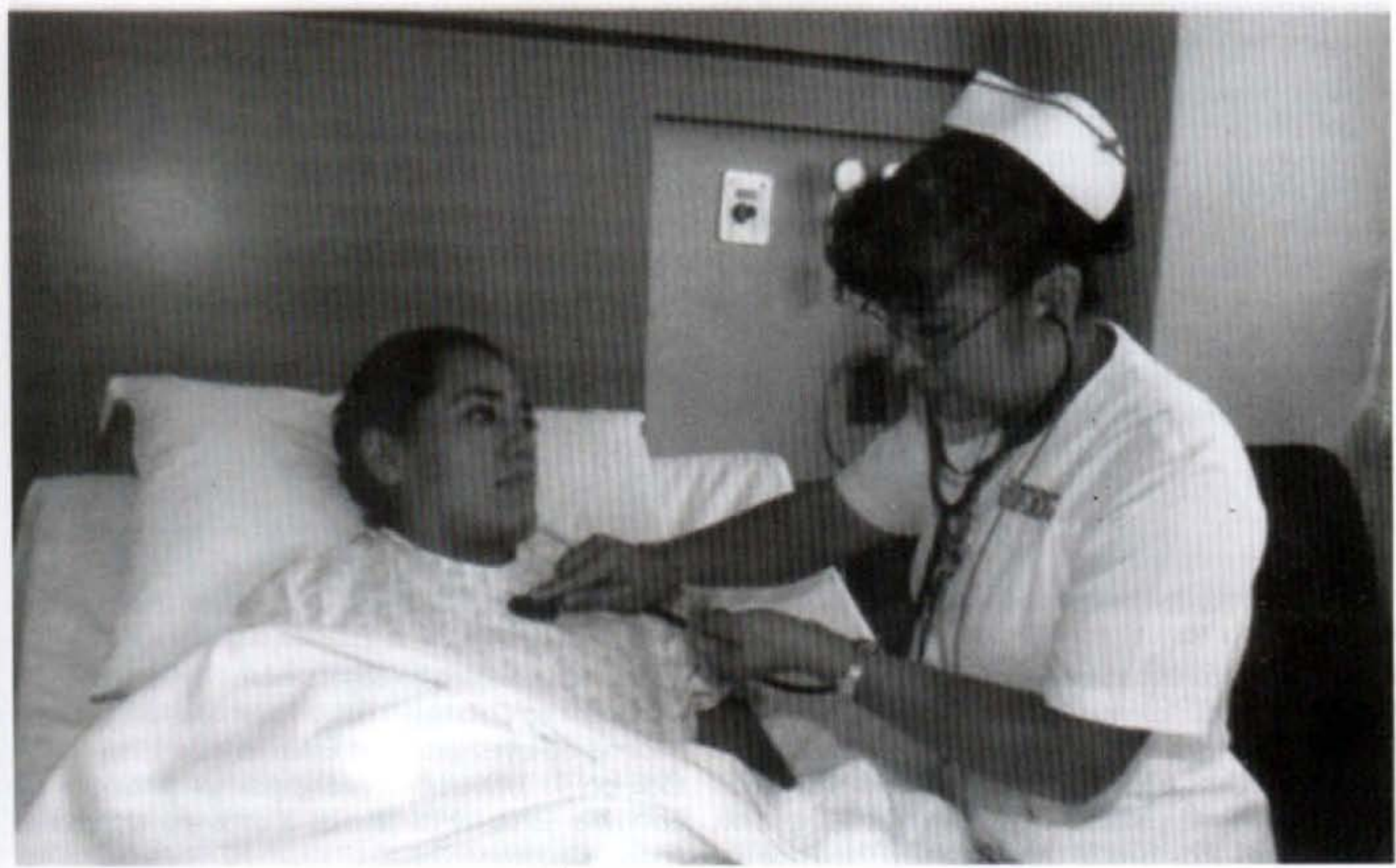

